\title{
Laser beams with conical refraction patterns
}

Yurii V. Loiko, Grigorii S. Sokolovskii, David Carnegie, Alex Turpin, Jordi Mompart, et al.

Yurii V. Loiko, Grigorii S. Sokolovskii, David Carnegie, Alex Turpin, Jordi Mompart, Edik U. Rafailov, "Laser beams with conical refraction patterns," Proc. SPIE 8960, Laser Resonators, Microresonators, and Beam Control XVI, 89601Q (4 March 2014); doi: 10.1117/12.2039893

SPIE. Event: SPIE LASE, 2014, San Francisco, California, United States 


\title{
Laser beams with conical refraction patterns
}

\author{
Yurii V. Loiko* ${ }^{1}$ Grigorii S. Sokolovskii ${ }^{2,1,3}$, David Carnegie ${ }^{1}$, Alex Turpin ${ }^{4}$, Jordi Mompart ${ }^{4}$, and \\ Edik. U. Rafailov ${ }^{1}$ \\ ${ }^{1}$ Photonics and Nanoscience Group, School of Engineering, Physics and Mathematics, University of \\ Dundee, Dundee DD1 4HN, UK \\ ${ }^{2}$ Ioffe Physico-Technical Institute, 26 Polytechnicheskaya str., St Petersburg, 194021, Russia \\ ${ }^{3}$ Petersburg State Electrotechnical University "LETI", St Petersburg, 195220, Russia \\ ${ }^{4}$ Departament de Física, Universitat Autònoma de Barcelona, E-08193, Bellaterra, Spain
}

\begin{abstract}
Laser beams with cone-refracted output from the plane mirror is demonstrated for the first time in lasers based on intracavity conical refraction (CR) phenomenon. Transverse profile of such lasers comprises a crescent ring of CR-like distribution, where any opposite points are of orthogonal linear polarizations. We confirm the existence of such mode of CR lasers by two observations. First, cascaded CR in reflection geometry has been demonstrated for first time and it provides experimental prove that a light beam passed along optic axis of a biaxial crystal, reflected back from a plane mirror and passed again through the crystal is restored. Second, CR cavity mode with CR-like pattern through the plane mirror is experimentally and theoretically demonstrated for the first time.
\end{abstract}

Keywords: conical refraction, laser, transverse profile, light polarization, conical refraction optical resonator

\section{INTRODUCTION}

Spatial polarization distribution, i.e. state of polarization (SOP), of laser beams is one of the key parameters served for numerous applications. SOP affects laser beam focusing. Tighter focusing of radially polarized beams with respect to the azimuthally polarized beams has been reported ${ }^{1}$, which is of particular interest in particle trapping and manipulation. Metal cutting depends on SOP in what laser beams with radial polarization distribution are more efficient ${ }^{2}$. In recent years light beams with conical refraction pattern attract attention due to potential applications, for instance, in particle trapping $^{3-6}$, as a beam converter ${ }^{7-9}$ as well as in free space optical communication ${ }^{10}$.

Any laser comprises an optical resonator and a gain medium. Isotropic optical resonators can maintain modes of any SOP. Finally, the SOP of output laser beam is defined by the linear anisotropies remnant or introduced to the optical resonator, by gain anisotropy and by anisotropy of intracavity absorption. Main part of lasers generates output beams with homogeneous (in transverse plane) distribution of either linearly or circularly SOP. Laser beams with azimuthal and radial distribution of SOP have been previously demonstrated as well ${ }^{11-16}$. Lasers that utilize conical refraction effect in active (gain) medium have been reported recently with hemispherical resonator design ${ }^{17,18}$. In conical refraction a ring field structure appears close to the focal plane. Any two opposite points of the ring are linearly orthogonally polarized and direction of linear polarization is continuously rotated along the ring. It has been experimentally reported that the output beam of CR laser from the plane mirror is linearly polarized ${ }^{17}$, while the output from the curved mirror was experimentally reported as being of CR-like transverse structure ${ }^{18}$.

Here further details are presented about spatio-polarization profiles that can be generated in CR lasers with plane output coupler and curved rear mirrors. In particular, we show that CR-like patterns are possible from the plane mirror and provide background for such observation. The paper is organized as follows. An introduction into the CR phenomenon based on recent findings is presented in Section 2 where, for the first time, we report cascaded conical refraction in reflection geometry, when an incident beam passes through biaxial crystal, reflects from a mirror and passes back through the same biaxial crystal. In Section 3 we focus on CR resonator mode. We present experimental demonstration and theoretical confirmation of CR resonator mode with CR-like transverse pattern from the plane mirror. In Section 4 we report CR-like structure from the plane mirror of CR laser. Finally, in Section 5 we summarize conclusions.

*y.loika@dundee.ac.uk; phone (+44) (0) 1382381076 


\section{CONICAL REFRACTION IN REFLECTION}

Previously conical refraction has been studied with light beams passing through biaxial crystals. Here we present results on CR in reflection geometry, when a light beam passes through a biaxial crystal then it is reflected from a mirror and passes back through the same biaxial crystal, which gives insight into pattern (mode) formation of a CR optical resonator. The latter one is thought as optical cavity with intracavity biaxial crystal where conical refraction phenomenon takes place.

\subsection{Cone model of conical refraction}

After Hamilton 1832, CR phenomenon was described as follows. A narrow beam that enters a biaxial crystal along one of the optic axes evolves as a hollow slanted cone, and on the exit surface of the crystal will refract as a hollow tube. Only recently ${ }^{19}$, it has been demonstrated that in the CR an input focused beam splits into two cones. According to this model, for an input uniformly polarized light beam with axially symmetric intensity distribution, such for instance, as $\mathrm{TEM}_{00}$ mode, the field behind the biaxial crystal can be represented as a sum of two CR cones $\vec{C}_{ \pm}\left(Z, \varphi_{\rho}, \rho, \rho_{0}\right)$ :

$$
\begin{array}{r}
\vec{E}\left(Z, \varphi_{\rho}, \rho, \rho_{0}\right)=\vec{C}_{+}\left(Z, \varphi_{\rho}, \rho, \rho_{0}\right)+\vec{C}_{-}\left(Z, \varphi_{\rho}, \rho, \rho_{0}\right), \\
\vec{C}_{ \pm}\left(Z, \varphi_{\rho}, \rho, \rho_{0}\right)=\sum_{s= \pm 1} A_{ \pm s}\left(Z, \rho, \rho_{0}\right) \vec{d}_{s}\left(\varphi_{\rho}\right)\left(\vec{d}_{s}\left(\varphi_{\rho}\right) \cdot \vec{d}_{i n}\right) \\
A_{q s}\left(Z, \rho, \rho_{0}\right)=\int_{0}^{\infty} d k k e^{-i Z k^{2} / 4} a(k) e^{i q \rho_{0} k}\left(J_{0}(k \rho)-i q s J_{1}(k \rho)\right) / 2
\end{array}
$$

where dot in Eq.(2) means scalar product of two vectors. $J_{k}(k \rho)$ denotes $\kappa$-th order Bessel function of the first kind. $\rho_{0}=R_{0} / w_{0}$ defines the CR ring radius at the Lloyd plane $R_{0}$ normalized to the waist radius of an input light beam $w_{0}$. $a(k)=\int_{0}^{\infty} \mathrm{d} \rho \rho J{ }_{0}(\rho k) E_{\text {in }}(\rho)$ represents radial dependence of 2D Fourier transform of an input beam with uniform polarization described by unit vector $d_{i n}$. Cylindrical coordinates $Z$ and $\rho$ are normalized to Rayleigh length and waist radius of the input beam, respectively. CR polarization basis is given by two unit vectors:

$$
\vec{d}_{+}(\varphi)=\{\cos (\varphi / 2), \sin (\varphi / 2)\}, \quad \vec{d}_{-}(\varphi)=\{\sin (\varphi / 2),-\cos (\varphi / 2)\} .
$$

In Fig. 1 we present an example of two CR cones in $(Z, \rho)$ plane for a circularly polarized input light beam.
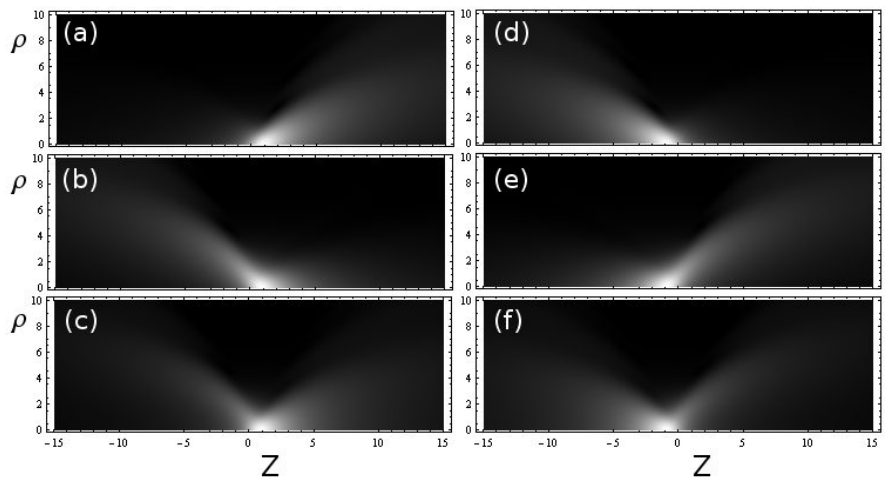

Figure 1. Decomposition of conically refracted beam into two cones, $\mathrm{C}_{+}$(left column) and $\mathrm{C}_{\text {. }}$ (right column), with amplitudes $\mathrm{A}_{+-}$(a) $\mathrm{A}_{++}$(b), $\mathrm{A}_{-}$(d) and $\mathrm{A}_{-+}$(e). Intensity distributions of respective cones are shown in figures (c) and (f). Input beam is of uniform circular polarization and biaxial crystal is characterized by $\rho_{0}=1$.

For a focused input light beam, the two cones or their continuations are shifted with respect to each other along the direction of propagation. $\mathrm{C}_{+}$cone (see Eq.(2) and left column in Fig.1) is focused after the C. cone (see right column in Fig.1). The centre (vertex) points of these cones have already been identified as the two bright points known as the Raman spots of conical refraction. Polarization distribution for each cone is switched at the Raman spot to orthogonal one, i.e. from $\mathrm{d}_{+}$to $\mathrm{d}_{-}$for $\mathrm{C}_{+}$cone (see Figs.1(a,b) and vise-versa for $\mathrm{C}_{-}$cone (see Figs.1(d,e). Therefore, the cones are of orthogonal polarization at each spatial point before the first Raman spot and after the second one. Between the Raman sports the two cones have the same spatial distribution of SOP. Therefore, the two cones cross each another and interfere 
in space between the Raman spots. It leads to a bright structure of double light rings known as the Poggendorff splitting. This structure appears at a plane known as the Lloyd plane and it is usually associated with CR phenomenon. For CR configuration with $\rho_{0}<1.06$ the Poggendorff splitting disappears and only one CR ring remains ${ }^{20}$ as demonstrated in Fig. 2. The symmetry axes of two cones are shifted in transverse direction with respect to the axis of an input beam. This shift (not shown in Fig. 2) is in the plane of the crystal optic axes and, in paraxial approximations, it is equal to the CR ring radius measured at the Lloyd plane.
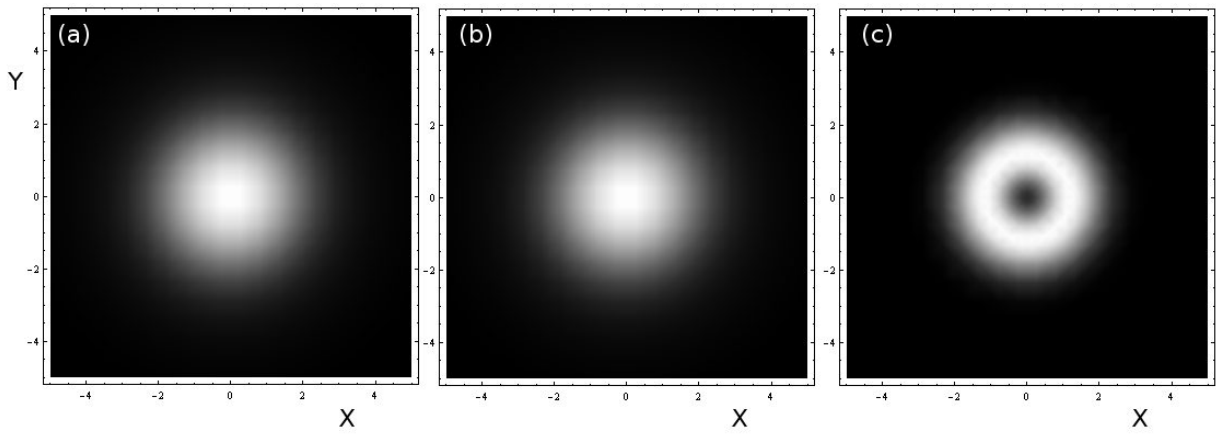

Figure 2. Transverse intensity pattern of $\mathrm{C}_{+}$(a) and $\mathrm{C}_{-}$(b) cone and their interference (c) at the Lloyd plane $(\mathrm{Z}=0)$ of the $\mathrm{CR}$ with an input beam of uniform circular polarization and $\rho_{0}=1$, i.e. for parameters of Fig. 1.

\subsection{Conical refraction with reflection from a mirror}

Optical cavities are usually formed by mirrors. Here we present results of conical refraction in reflected configuration, i.e. when a light beam passes through a biaxial crystal and it is reflected by a mirror behind the crystal to pass back. This provides a step to understanding of mode formation in CR optical resonator. Our setup is shown in Fig. 3. An input linearly polarized light beam $\left(980 \mathrm{~nm}\right.$ with $\mathrm{M}^{2} \sim 10$ from an electrically pumped (EP) VECSEL) passes through a nonpolarizing beam splitter (NP BS). It is focused by a lens L into a biaxial crystal (BC). The beam undergoes conical refraction in forward direction inside the crystal. After reflection from a mirror $\mathrm{M}$ the beam passes in backward direction through the same system of crystal and lens. The backward (output) beam redirected by the NP BS is registered by a CCD camera.

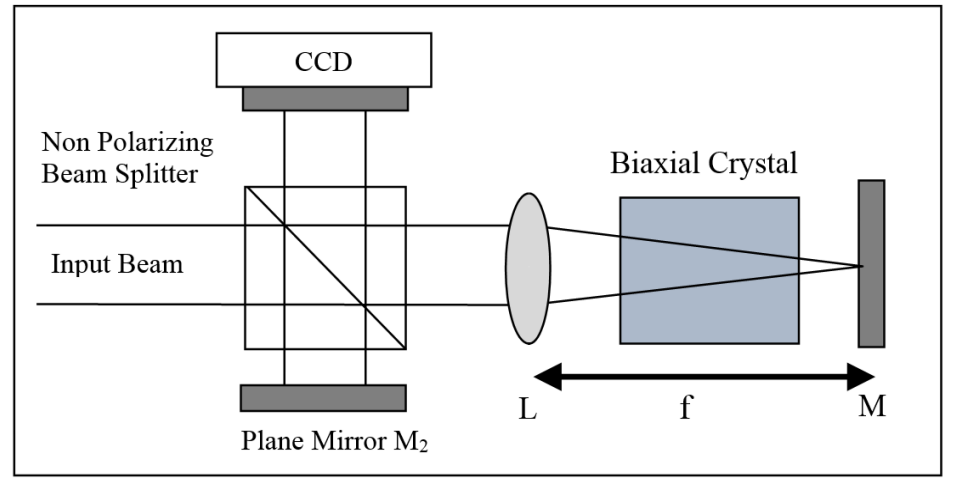

Figure 3. Experimental setup for conical refraction in reflection geometry, i.e. when an input beam focused by a lens L

(focal length $\mathrm{f}$ ), passes through a biaxial crystal, reflects from a mirror $\mathrm{M}$ and passes in backward direction through the same system of biaxial crystal and lens. Non-polarizing beam splitter is served to view the backward output beam and initial beam reflected from a plane mirror $\mathrm{M}_{2}$.

An input beam undergoes conical refraction when it propagates in forward direction through the crystal. CR ring structure is formed at the Lloyd plane, see Fig. 4(c). When plane mirror is used as a reflector $M$ and its plane surface coincides exactly with the Lloyd plane, the input beam is restored in backward direction after the crystal, see Figs. 4(a) and (b). When the plane mirror surface is misaligned from the Lloyd plane the input beam is no longer restored in backward direction. In Figs. 4(d,e) transverse intensity pattern of backward beam is shown when the plane mirror surface is angularly misaligned with respect to the Lloyd plane in horizontal (Fig. 4(d)), and vertical (Fig. 4(e)) direction. Two local minima and a crescent-like part of a ring structure appear in the backward beam for small angular misalignment. 
For misalignment of plane mirror surface in horizontal (vertical) direction, see Figs. 4(d) and 4(e), the mentioned minima belong to a vertical (horizontal) line, while crescent-like pattern appear in the direction of misalignment.

When a curved mirror is used as the reflector M (see Fig.3) the initial beam structure is no longer restored even when the focal points of the focusing lens and mirror coincide. The backward beam has in general CR structure of two cones.
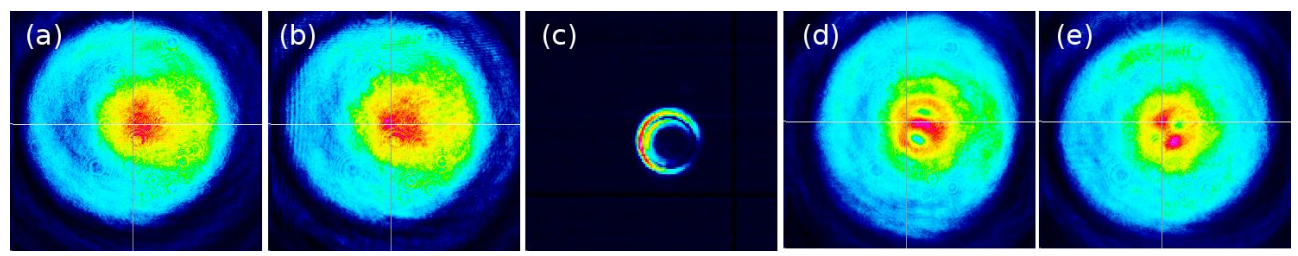

Figure 4. Input light beam from an EP VECSEL 980nm (a), its reconstruction in backward direction (b) in cascaded CR of Figure 3 with a plane mirror M. CR ring (c) imaged in forward direction from the plane mirror $\mathrm{M}$ by an additional lens (not shown in figure 3). Leftmost (rightmost) point of the CR ring is of linear vertical (horizontal) polarization. Nd:KGW biaxial crystal of length $6 \mathrm{~mm}$ was used in the setup. It provides CR ring with diameter $\varnothing=204 \mu \mathrm{m}$ at $980 \mathrm{~nm}$. Focal length of lens is equal to $\mathrm{f}=75 \mathrm{~mm}$. Beam reconstructed in backward direction, when the mirror surface is angularly misaligned in horizontal (d) and in vertical (e) direction with respect to the Lloyd plane of conical refraction.

\section{CONICAL REFRACTION OPTICAL CAVITY}

Now we consider an optical cavity with intracavity biaxial crystal and show that CR-like structure of the field can be observed through the plane mirror. The setup is shown in Fig.5. The CR optical cavity comprises a biaxial crystal with one of its' optic axes aligned with optical axis of the resonator formed by plane and curved mirrors $\mathrm{M}_{1,2}$. A glass plate (GP) inclined to the optical axis of the CR cavity is used in order to inject light from an external laser source. We used $980 \mathrm{~nm}$ output from EP VECSEL. Two configurations have been explored with plane $\mathrm{M}_{1}$ and curved $\mathrm{M}_{2}$ mirrors in the first configuration and vise-versa in the second one. Identical results have been obtained for both configurations, which confirm independence of the observed CR resonator mode on the position of injected light, i.e. it does not depend on whether light is injected close to the plane or to the curved mirror.

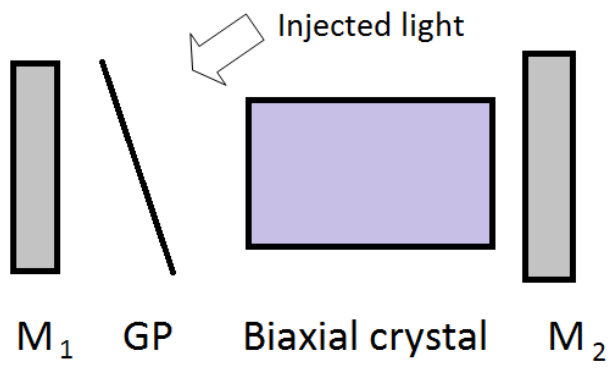

Figure 5. Experimental setup for observation of CR resonator modes. Glass plate (GP) is used in order to inject light from an external laser source. Two configurations have been explored with plane mirror $\mathrm{M}_{1}$ and curved $\mathrm{M}_{2}$ mirror for the first configuration and vise-versa for the second one.
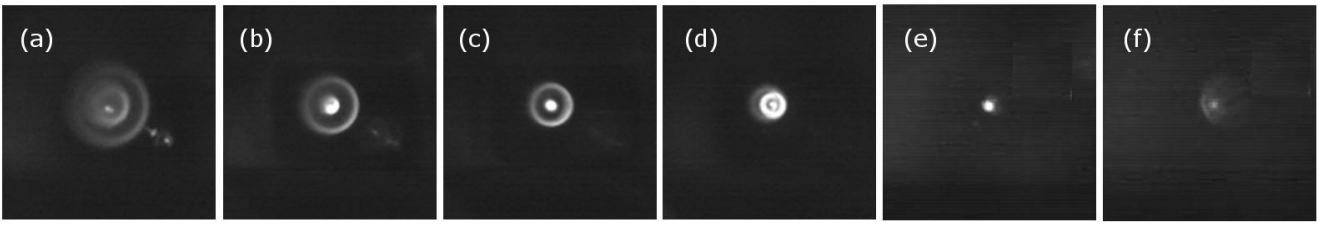

Figure 6. Mode of CR optical resonator, i.e. isotropic optical cavity with axis aligned along optic axis of intracavity biaxial crystal. The resonator comprises plane and concave (radius of curvature of $75 \mathrm{~mm}$ ) mirrors shown in Fig.5. Output beam propagating from the plane (a-d) and curved (e,f) mirrors. The mode of CR optical cavity includes central spot at the curved mirror (e) as well as central spot and ring at the plane mirror (d). Figures (d), (c), (b) and (a) show cones' divergence from the plane mirror. Nd:KGW intracavity biaxial crystal of length $22 \mathrm{~mm}$ was used with $\mathrm{CR}$ ring radius of $\mathrm{R}_{0}=374 \mu \mathrm{m}$.

Distance of $125 \mathrm{~mm}$ between the plane and curved (radius of curvature $200 \mathrm{~mm}$ ) mirrors provides $\mathrm{w}_{0}=181 \mu \mathrm{m}\left(\rho_{0}=2.06\right)$. Additional small spots outside the rings in figures $(a-c)$ correspond to reflection of injected light from crystal edge. 
We were able to excite CR cavity mode with CR-like field structure at the plane mirror as shown in Fig.6(d). This structure possesses bright central spot and ring which are formed by two cones. Their divergence out from the plane mirror is demonstrated by sequence of Figs. 6(d), (c), (b) and (a). For the CR cavity mode excited in this experiment we observed only one bright spot through the curved mirror (see Figs.6(e,f)).

We performed calculation of $\mathrm{CR}$ resonator mode on the basis of beam propagation method and previous theoretical models for conical refraction ${ }^{19-22}$, see also Eqs.(1-3) above. Theoretical results are shown in Fig.7. They corroborate with experimentally measured structure observed through the plane and curved mirror, see Figs.6(d,e) and Figs.7(a,b), respectively.

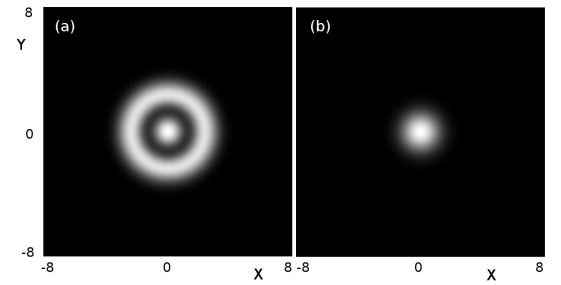

Figure 7. Enlarged pattern of CR resonator mode at plane (a) and curved (b) mirror calculated numerically with parameters of biaxial crystal and optical cavity as given in Fig.6.

\section{CONICAL REFRACTION PATTERN FROM PLANE MIRROR OF CR LASER}

In this section we demonstrate CR-like pattern from plane mirror of CR laser. As an active element of CR laser we used a Nd:KGd $\left(\mathrm{WO}_{4}\right)_{2}$ crystal with dimensions of $3 \times 4 \times 12 \mathrm{~mm}^{3}$. The volume concentration of the $\mathrm{Nd}^{3+}$ ions was $1.9 \times 10^{20} \mathrm{~cm}^{-3}$ ( 3 at. \% doping). The polished plane parallel surfaces of the crystal were antireflection (AR) coated at pump (805 nm) and laser $(1067 \mathrm{~nm})$ wavelengths. They are perpendicular to one of the crystal optic axes with angular misalignment better than $1^{\circ}$. The conicity (semiangle of internal conical refraction) of Nd:KGW crystal is $17 \mathrm{mrad}$, which results in CR ring radius of $\mathrm{R}_{0}=204 \mu \mathrm{m}$ for our sample.

The CR laser cavity was the same as shown in Fig.5 (but without the glass plate GP) with plane mirror used as an output coupler (3\% transmission at laser wavelength) and concave rear mirror (100 $\mathrm{mm}$ radius of curvature). The crystal was mounted in a metallic holder without extra cooling. The laser was end-pumped through the rear mirror by an unpolarised, multimode (core diameter of $200 \mu \mathrm{m}, \mathrm{M}^{2}=40$ ) fiber coupled diode laser at a wavelength around $805 \mathrm{~nm}$ focused into the crystal. The waist radius of the focused pump beam was $200 \mu \mathrm{m}$, which means 2 x magnification from the fibre.

The alignment procedure here is slightly different from that reported in previous publications ${ }^{17,18}$. The pump beam has been focused to the crystal centre. It has been aligned to one of respective crystal optic axes by observing CR ring at the Lloyd plane of transmitted light beam (see Figs.8(b,c)). The structure of a beam passing along optic axis of an absorptive biaxial crystal is shown in Fig. 8(a). Two Raman spots are almost undistinguishable (elongated along propagation direction). However, $\mathrm{CR}$ ring structure at the Lloyd plane remains clearly visible for small values of $\mathrm{M}^{2}$ factor (see Fig.8(b)). The larger the $\mathrm{M}^{2}$ factor of the input beam the thicker the CR ring at the Lloyd plane. Consequently, it is difficult to observe the $\mathrm{CR}$ ring at the Lloyd plane for pump beams with large $\mathrm{M}^{2}$ factor, see Fig.8(c), and for pump geometry when the pump beam radius becomes two times larger than the $\mathrm{CR}$ ring radius.

Above the laser threshold, axis of optical cavity formed by a plane and curved mirror is aligned close to one of the crystal optic axes at laser wavelength. Crescent-like intensity pattern as shown in Fig.9(a) appears when the optical cavity axis and crystal optic axis are close to each other. In order to confirm CR-like structure, SOP is measured by placing a polarizer in front of the CCD camera. Intensity patterns recorded by the CCD camera with polarizer rotated by a certain angle are shown in Figs.9(b-e). They confirm CR-like polarization distribution of the observed CR laser mode. When the pump beam is focused close to the crystal surface oriented to the curved mirror and CR optical cavity is oriented along the optic axis at laser wavelength the ring structure observed through the plane mirror disappears and central bright spot remains only, which corroborates with previously reported experimental results ${ }^{17,18}$. While in previous experiments high laser efficiency has been reported ${ }^{17}$, we have achieved only $30 \%$ as maximum for optical-to-optical efficiency for the CR-like mode and pumping scheme reported in this paper, which is similar to the results reported in 
Ref. $^{18}$. Further publications will be devoted to resolving complete structure of CR laser modes and their lasing conditions.

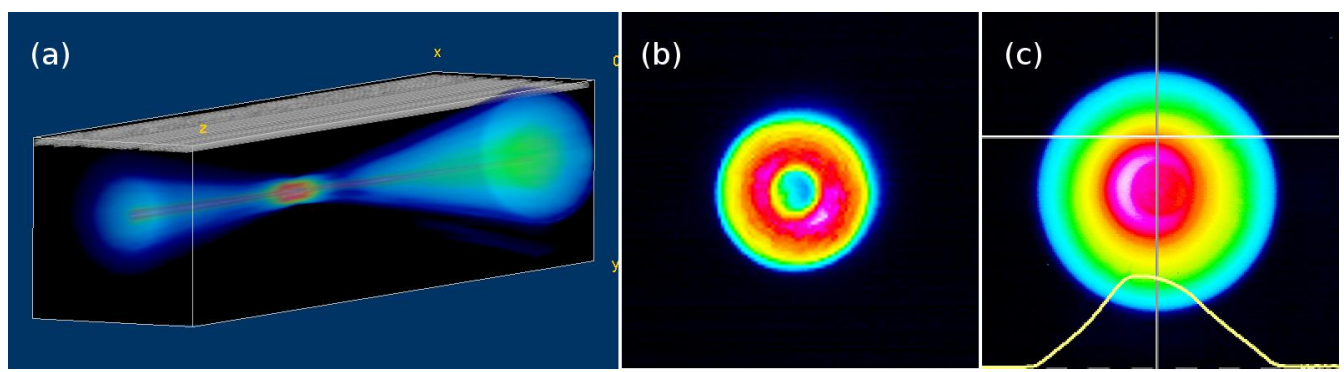

Figure 8. 3D image (a) of an optical field passed through an absorptive biaxial crystal along one of the crystal optic axes. Input beam with waist radius of $200 \mu \mathrm{m}$ has been used. $3 \% \mathrm{Nd}$ doped $\mathrm{KGW}$ crystal of length $12 \mathrm{~mm}$ used as a biaxial crystal provides normalized CR ring radius of $\rho_{0}\left(\lambda_{\text {Pump }}\right) \sim 1$, which corresponds to Fig.2. 2D pattern of CR ring structure at the Lloyd plane for an input beam with $\mathrm{M}^{2}$ of 24 (b) and 40 (c).
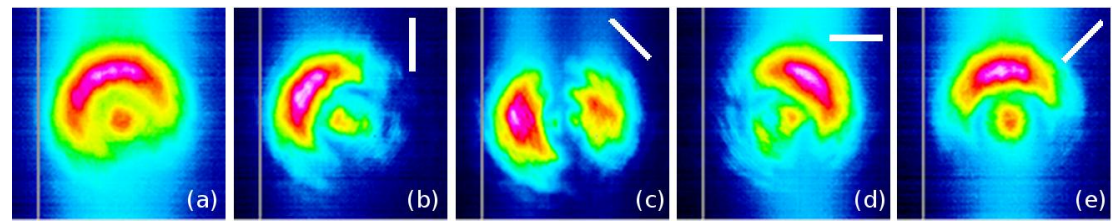

Figure 9. CR-like pattern observed through the plane mirror of CR laser at a CCD camera without (a) and with polarizer (be) at the front of the camera rotated counter-clock wise from vertical direction by step of $\pi / 4$. Thick white line in figures (be) shows polarizer orientation. CR laser was formed by a Nd:KGW biaxial crystal of length $\mathrm{L}_{\mathrm{BC}}=12 \mathrm{~mm}\left(\mathrm{R}_{0}=204 \mu \mathrm{m}\right)$ placed between plane output coupler (transmission of $\mathrm{T}=3 \%$ ) and curved rear mirror (AR at pump wavelength of $805 \mathrm{~nm}$, radius of curvature $\mathrm{R}=100 \mathrm{~mm}$ ) separated by a distance of $90 \mathrm{~mm}\left(\rho_{0}=2.06\right)$, which corresponds to the CR resonator mode of Figs. 6 and 7.

\section{CONCLUSIONS}

We have investigated cascaded conical refraction in reflection geometry, i.e. when a light beam undergoes conical refraction in a biaxial crystal and then reflects from a mirror placed behind the crystal to pass back through the same crystal. We demonstrate for the first time that initial beam is restored in such cascaded CR when the reflective mirror is a plane mirror. The backward beam has CR-like structure when reflection occurs from a curved mirror. This provides insight into formation of CR optical resonator modes. CR cavity mode with CR-like pattern through the plane mirror is experimentally and theoretically demonstrated for the first time.

Finally, laser beams with cone-refracted output from the plane mirror is demonstrated for the first time in lasers based on intracavity CR. Transverse profile of such lasers comprises a crescent ring of CR-like structure. We present experimental results for $\mathrm{CR}$ lasers with $\mathrm{Nd}$-doped $\mathrm{KGd}\left(\mathrm{WO}_{4}\right)_{2}$ biaxial crystals used as an active laser medium and with hemispherical design of optical cavity. This opens new opportunities for generation of CR-like laser beams.

\section{Acknowledgments}

The research leading to the results presented here has received funding from the European Union's Seventh Framework Programme (FP7/2007-2013) managed by the Research Executive Agency (http://ec.europa.eu/rea) under grant agreement no. 314936 FP7-SME-2012. 


\section{REFERENCES}

[1] Quabis, S., Dorn, R., Eberler, M., Glöckl, O., and Leuchs, G., "Focusing light to a tighter spot," Opt. Commun. 179, 1 (2000).

[2] Niziev, V. G. and Nesterov, A. V., "Influence of beam polarization on laser cutting efficiency," J. Phys. D: Appl. Phys. 32, 1455-1461 (1999).

[3] Turpin, A., Shvedov, V., Hnatovsky, C., Loiko, Yu. V., Mompart, J. and Krolikowski, W., "Optical vault: a reconfigurable bottle beam based on conical refraction of light," Optics Express 21(22), 26335-26340 (2013).

[4] O’Dwyer, D. P., Ballantine, K. E., Phelan, C. F., Lunney, J. G., and Donegan, J. F., "Optical trapping using cascade conical refraction of light," Optics Express 20(19), 21119-21125 (2012).

[5] O’Dwyer, D. P., Phelan, C. F., Ballantine, K. E., Rakovich, Y. P., Lunney, J. G., and Donegan, J. F., "Conical diffraction of linearly polarised light controls the angular position of a microscopic object," Optics Express 18 (26), 27319-27326 (2010).

[6] McDougall, C., Henderson, R., Carnegie, D. J., Sokolovskii, G. S., Rafailov, E. U., and McGloin, D., "Flexible particle manipulation techniques with conical refraction based optical tweezers," SPIE Proc. Vol. 8458, 845824 (2012).

[7] Peet, V., "Biaxial crystal as a versatile mode converter," J. Optics 12(9), 095706 (2010).

[8] Stepanov, M. A., "Transformation of Bessel beams under internal conical refraction," Opt. Commun. 212 (1-3), 11-16 (2002).

[9] King, T. A., Hogervorst, W., Kazak, N. S., Khilo, N. A., and Ryzhevich, A. A., "Formation of higher-order Bessel light beams in biaxial crystals," Opt. Commun. 187 (4-6), 407-414 (2001). Kazak, N. S., Khilo, N. A., and Ryzhevich, A. A., "Generation of Bessel light beams under the conditions of internal conical refraction," Quantum Electron. 29 (11), 1020-1024 (1999).

[10] Turpin, A., Loiko, Yu.V., Kalkandjiev, T. K., and Mompart, J., "Free space optical polarization de-multiplexing and multiplexing by means of conical refraction," Opt. Lett. 37 (20), 4197-4199 (2012). Loiko, Yu. V., Turpin, A., Kalkandjiev, T. K., and Mompart, J., "Conical refraction multiplexing for free space optical communications," Proc. SPIE 8246, 82460T (2012).

[11] Mushiake, Y., Matsumura, K., and Nakajima, N., "Generation of radially polarized optical beam mode by laser oscillation," Proc. IEEE Lett. 60 (9), 1107-1109 (1972).

[12] Pohl, D., "Operation of a ruby laser in the purely transverse electric mode TE ${ }_{01}$, " Appl. Phys. Lett., 20 (7), 266267 (1972)

[13] Oron, R., Blit, S., Davidson, N., Friesem, A.A., Bomzon, Z., and Hasman, E., "The formation of laser beams with pure azimuthal or radial polarization," Appl. Phys. Lett. 77, 3322 (2000).

[14] Nesterov, A.V., Niziev, V.G., and Yakunin, V.P., "Generation of high-power radially polarized beam," J. Phys. D: Appl. Phys. 32, 2871-2875 (1999).

[15] Moser, T., et al. "Polarization-selective grating mirrors used in the generation of radial polarization," Appl. Phys. B 80, 707-713 (2005).

[16] Kozawa, Y., and Sato, S., "Generation of a radially polarized laser beam by use of a conical Brewster prism," Opt. Lett. 30 (22), 3063-3065 (2005).

[17] Abdolvand, A., Wilcox, K. G., Kalkandjiev, T. K., and Rafailov, E. U., "Conical refraction Nd:KGd($\left(\mathrm{WO}_{4}\right)_{2}$ laser," Opt. Express 18, 2753-2759 (2010).

[18] Wilcox, K. G., Abdolvand, A., Kalkandjiev, T. K., and Rafailov, E. U., "Laser with simultaneous Gaussian and conical refraction outputs," Appl. Phys. B 99, 619-622 (2010).

[19] Sokolovskii, G. S., Carnegie, D. J., Kalkandjiev, T. K., and Rafailov, E. U., "Conical Refraction: New observations and a dual cone model," Optics Express 21, 11125-11131 (2013).

[20]Loiko, Yu. V., Turpin, A., Kalkandjiev, T. K., Rafailov, E. U., and Mompart, J., "Generating a three dimensional dark focus from a single conically refracted light beam," Optics Letters 38(22), 4648-4651 (2013).

[21]Belskii, A. M., and Khapalyuk, A. P. "Internal conical refraction of bounded light beams in biaxial crystals," Opt. Spectrosc. (USSR) 44, 436-439 (1978).

[22] Berry, M. V. and Jeffrey, M. R., "Conical diffraction: Hamilton's diabolical point at the heart of crystal optics," Prog. Opt. 50, 13-50 (2007). 